\title{
NEW USES OF WESTPHAL CONDENSATION: SYNTHESIS OF FLAVOCORYLENE AND RELATED INDOLO[2,3-a]QUINOLIZINIUM SALTS.
}

\author{
María P. Matia ${ }^{\mathrm{a}}$, Jesús Ezquerra ${ }^{\mathrm{b}}$, José L. García-Navióa, \\ Juan J. Vaquero ${ }^{\mathbf{a}}$ and Julio Alvarez-Builla*a. \\ a Departamento de Quimica Orgánica, Universidad de \\ Alcalá, 28871 Alcalá de Henares, Madrid (Spain) \\ b Centro de Investigación LILLY S.A., Paraje de la Cruz \\ s/n, 28130 Valdeolmos, Madrid (Spain)
}

\begin{abstract}
Using the Westphal condensation, flavocorylene and related Indolo[2,3-a]quinolizinium salts have been prepared in two steps, starting from commercially available $\mathrm{B}$-carboline derivatives.
\end{abstract}

The small group of biogenetically-interesting ${ }^{1}$ indole alkaloids that incorporate the zwitterionic indolo[2,3-a]quinolizinium ring system $\mathbf{I}$ has received limited attention in the synthesis field. ${ }^{2}$ Some of its representatives such as flavopereirine (1) and sempervirine (2) have been described to possess antitumour activity, ${ }^{3}$ but the relatively complex preparation methods has reduced the availability of analogs.

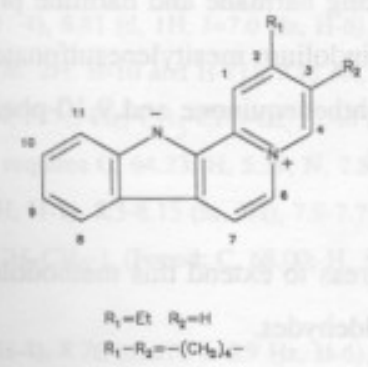

Our interest in the Westphal condensation ${ }^{4}$ as an easy way to prepare quinolizinium salts ${ }^{5}$ led us to exploit the reactivity of $9 \mathrm{H}-2$-ethoxycarbonylmethyl-1-methylpyrido[3,4-b]indolium bromides $\mathbf{4}$ and $\mathbf{5}$, which were prepared in high yields from commercially available B-carbolines harmane and harmine. ${ }^{6}$ 
Basic condensation of both salts with symmetric 1,2-diketones, such as 3,4-hexanedione and 1,2acenaphthenequinone, provided the $12 \mathrm{H}$-indolo[2,3-a]quinolizinium bromides ${ }^{7} \mathbf{8 a}, \mathbf{b}$ and $\mathbf{9 a}, \mathbf{b}$. Anion exchange of bromide $(8 \mathrm{a}, \mathrm{X}=\mathrm{Br})$ produced the already described Flavocorylene hydrochloride ${ }^{8}$.

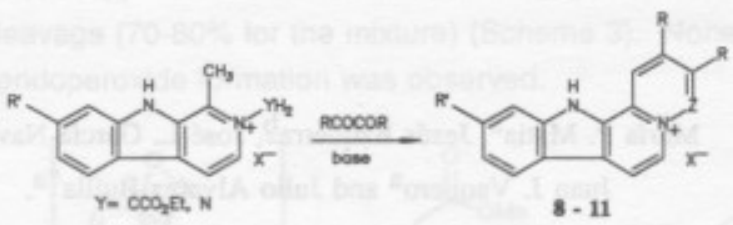

\begin{tabular}{|c|c|c|c|c|c|c|}
\hline mpound & $\mathbf{R}^{*}$ & z & $\mathrm{x}$ & $\mathbf{R}$ & $\mathbf{R}$ & Yield(\%) \\
\hline & & & & & & \\
\hline $8 \mathrm{a}$ & H & $\mathrm{CH}$ & $\mathrm{Br}$ & $\mathrm{CH}_{3} \mathrm{CH}_{2}$ & $\mathrm{CH}_{3} \mathrm{CH}_{2}$ & 60 \\
\hline $8 b$ & H & $\mathrm{CCO}_{2} \mathrm{Et}$ & $\mathrm{Br}$ & \multicolumn{2}{|c|}{ 1,8-Naphthalenediyl } & 94 \\
\hline $9 a$ & $\mathrm{OCH}_{3}$ & $\mathrm{CH}$ & $\mathrm{Br}$ & $\mathrm{CH}_{3} \mathrm{CH}_{2}$ & $\mathrm{CH}_{3} \mathrm{CH}_{2}$ & 50 \\
\hline $9 b$ & $\mathrm{OCH}_{3}$ & $\mathrm{CCO}_{2} \mathrm{Et}$ & $\mathrm{Br}$ & \multicolumn{2}{|c|}{ 1,8-Naphthalenediyl } & 70 \\
\hline 10b & $\mathrm{H}$ & $\mathrm{N}$ & MSTS* & \multicolumn{2}{|c|}{ 1,8-Naphthalenediyl } & 73 \\
\hline $10 c$ & H & $\mathrm{N}$ & MSTS & \multicolumn{2}{|c|}{ Diphen- $0 . \sigma^{\prime}$ - diyl } & 84 \\
\hline $11 b$ & $\mathrm{OCH}_{3}$ & N & MSTS & \multicolumn{2}{|c|}{ 1,8-Naphthalenediy! } & 75 \\
\hline $11 \mathrm{c}$ & H & N & MSTS & \multicolumn{2}{|c|}{ Diphen- $\sigma, \rho^{\circ}$-diyl } & 70 \\
\hline
\end{tabular}

- Mesitylensulfonate

Our methodology is equally applicable to the synthesis of the new $12 \mathrm{H}$-pyridazino[ $\left.2^{\prime}: 3^{\prime}, 1,2\right]$ pyrido[3,4-b]indolium mesitylenesulfonates $10 \mathrm{~b}, \mathrm{c}$ and $11 \mathrm{~b}, \mathrm{c}$ isoelectronic with the bromides 8 and 9 , by a modified Westphal condensation, recently developed by us. ${ }^{9}$ Thus, amination with $\mathrm{O}$-mesitylenesulfonylhydroxylamine $(\mathrm{MSH})^{10}$ of the starting harmane and harmine precursors yielded the corresponding 2-amino-1-methyl-9H-pyrido[3,4-b]-indolium mesitylenesulfonates 6 and 7 (X=MSTS) which, in basic conditions $^{8}$, reacted with 1,2-acenaphthenequinone and 9,10-phenanthrenequinone yielding the new salts 10 and 11 (X=MSTS).

Further experiments are in progress to extend this methodology to other 1,2-dicarbonyl compounds as asymmetric diketones and 1,2-ketoaldehydes.

In summary, the new approach represents a straightforward preparation of $12 \mathrm{H}$-indolo[2,3-a]quinolizinium derivatives which can be easily extended to new biologically interesting compounds. 


\section{References and notes.}

1. (a) Scott, A.I. Bioorg. Chem. 1974, 3, 398; (b) Kan-Fan, C; Husson, H.-P. Tetrahedron Lett. 1980, $21,4265$.

2. For recent synthetic efforts and references to the isolation of these alkaloids, see a) Teuber, H.J.; Quintanilla-Licea, R; Raabe, T. Liebigs Ann. Chem. 1988, 1111; (b) Gribble, G.W.; Johnson, D.A. Tetrahedron Letl. 1987, 44, 5259; c) Ninomiya, I.; Tada, Y.; Kiguchi, T; Yamamoto, O.; Naito, T. J. Chem. Soc. Perkin Trans. I 1984, 2035; d) Giri, V.S.; Maiti, B.C,: Pakrashi, S.C. Heterocycles 1984, 22, 233.

3. (a) Beljanski, M; Beljanki, M.S. Expl. Cell Biol. 1982, 50, 79; (b) Beljanski, M; Beljanki, M.S. Oncology. 1986, $43,198$.

4. Westphal, O.; Jahn, K.; Heffe, W. Arch. Pharm. 1961, $294,37$.

5. Matia, M.P.; Sanchez-Ferrando, F; Ezquerra, J; Garcia-Navio, J.L.; Vaquero, J J.; Alvarez-Builla, J. Tetrahedron. $1991,47$. 0000 .

6. ${ }^{1}$ H NMR spectra were recorded on a Varian Unity 300 instrument. Chemical shifts are expressed in parts per million downfield from tetramethylsilane. The proton resonances for the mesitylenesulfonate anion in $6,7,10 b, c$ and $11 b, c$ have not been listed as they are independent of the heterocyclic cation, signals appearing in all cases at $6.62 \mathrm{ppm}$ for the aromatic protons and 2.44 and $2.15 \mathrm{ppm}$ for the ortho and para methyl groups, respectively. Satisfactory microanalyses were obtained for all new compounds described, within $0.4 \%$ error.

Typical procedure: Equivalent amounts (10 mmol) of the corresponding heterocyclic precursor and ethyl bromcacetate in dry acetone $(30 \mathrm{ml})$ were refluxed for 4 hours. The precipitate was collected and recrystallized from ethanol.

Compound 4. $\left(250-252^{\circ} \mathrm{C}, 90 \%\right)^{1}{ }^{1} \mathrm{H}$ NMR(DMSO-d 6 ) $813.00(\mathrm{~s}, 1 \mathrm{H}, \mathrm{NH}), 8.75(\mathrm{~d}, 1 \mathrm{H}, \mathrm{J}=6.6 \mathrm{~Hz}, \mathrm{H}-3), 8.60$ (d, $1 \mathrm{H}$, $\mathrm{J}=6.6 \mathrm{~Hz}, \mathrm{H}-4), 8.45(\mathrm{~d}, 1 \mathrm{H}, \mathrm{J}=8.0 \mathrm{~Hz}, \mathrm{H}-5), 7.85-7.75(\mathrm{~m}, 2 \mathrm{H}, \mathrm{H}-7$ and H-8), 7.5-7.4 (m, 1H, H-6), $5.81(\mathrm{~s}, 2 \mathrm{H}$, $\mathrm{CH}_{2} \mathrm{CO}$ ), 4.25 (q, $2 \mathrm{H}, \mathrm{J}=7.1 \mathrm{~Hz},-\mathrm{CH}_{2} \mathrm{CH}_{3}$ ), 3.04 (s, 3H, $\mathrm{CH}_{3}-\mathrm{Cl}$ ), 1.25 (t, 3H, J=7.1 Hz, $\mathrm{CH}_{2} \mathrm{CH}_{3}$ ). (Found: C, 55.10 ; $\mathrm{H}, 5.15 ; \mathrm{N}, 7.95, \mathrm{C}_{16} \mathrm{H}_{17} \mathrm{BrN}_{2} \mathrm{O}_{2}$ requires $\mathrm{C}, 55.02 ; \mathrm{H}, 4.91 ; \mathrm{N}, 8.02$ ).

Compound 5. $\left(223-225^{\circ} \mathrm{C}, 80 \%\right){ }^{1} \mathrm{H}$ NMR(DMSO-d 6$) 812.82(\mathrm{~s}, 1 \mathrm{H}, \mathrm{NH}), 8.6-8.5(\mathrm{~m}, 2 \mathrm{H}, \mathrm{H}-3$ and H-4), $8.31(\mathrm{~d}, 1 \mathrm{H}$, $\mathrm{J}=8.7 \mathrm{~Hz}, \mathrm{H}-5), 7.13(\mathrm{~d}, 1 \mathrm{H}, \mathrm{J}=1.9 \mathrm{~Hz}, \mathrm{H}-8), 7.06(\mathrm{dd}, 1 \mathrm{H}, \mathrm{J}=8.8$ and $1.9 \mathrm{~Hz}, \mathrm{H}-6), 5.72\left(\mathrm{~s}, 2 \mathrm{H},-\mathrm{CH}_{2} \mathrm{CO}-\right)_{1} 4.24(\mathrm{q}, 2 \mathrm{H}$, $\left.\mathrm{J}=7.1 \mathrm{~Hz},-\mathrm{CH}_{2} \mathrm{CH}_{3}\right), 3.94\left(\mathrm{~s}, 3 \mathrm{H},-\mathrm{OCH}_{3}\right), 2.98\left(\mathrm{~s}, 3 \mathrm{H}, \mathrm{CH}_{3}-\mathrm{Cl}\right), 1.26\left(\mathrm{~L}, 3 \mathrm{H}, \mathrm{J}=7.1 \mathrm{~Hz}, \mathrm{CH}_{2} \mathrm{CH}_{3}\right.$ ). (Found: $\mathrm{C}, 53,70 ; \mathrm{H}$, $5.20 ; \mathrm{N}, 7.55, \mathrm{C}_{17} \mathrm{H}_{19} \mathrm{BrN}_{2} \mathrm{O}_{3}$ requires $\left.\mathrm{C}, 53.83 ; \mathrm{H}, 5.05 ; \mathrm{N}, 7.39\right)$.

7. General Procedure: Equivalent amounts $(10 \mathrm{mmol})$ of the azinium salts $4-7$, the dicarbonyl derivative, and anhydrous sodium acetate $(0.82 \mathrm{~g}, 10 \mathrm{mmol})$ were suspended in dry acetone $(10 \mathrm{ml})$. The mixture was refluxed for $2 \mathrm{~h}$. The precipitate was filtered. Crystallization from the acetic acid/acetone yielded the compounds 8-11 in analytical grade. All melted above $260^{\circ} \mathrm{C}$.

8a. ${ }^{1} \mathrm{H}$ NMR(CD 3 OD) 89.05 (s, $\left.1 \mathrm{H}, \mathrm{H}-4\right), 8.81$ (d, $\left.1 \mathrm{H}, \mathrm{J}=7.0 \mathrm{~Hz}, \mathrm{H}-6\right), 8.63$ (s, $\left.1 \mathrm{H}, \mathrm{H}-1\right), 8.55(\mathrm{~d}, 1 \mathrm{H}, \mathrm{J}=7.0 \mathrm{~Hz}, \mathrm{H}-7)$, $8.32(\mathrm{~d}, 1 \mathrm{H}, \mathrm{J}=8.0 \mathrm{~Hz}, \mathrm{H}-8), 7.8-7.65(\mathrm{~m}, 2 \mathrm{H}, \mathrm{H}-10$ and $\mathrm{H}-11), 7.45(\mathrm{bt}, 1 \mathrm{H}, \mathrm{H}-9), 3.09\left(\mathrm{q}, 2 \mathrm{H}, \mathrm{J}=7.3 \mathrm{~Hz}, \mathrm{CH}_{2}-\mathrm{C}_{2}\right), 2.99$ (q, $2 \mathrm{H}, \mathrm{J}=7.3 \mathrm{~Hz}, \mathrm{CH}_{2}-\mathrm{C} 3$ ), $1.55\left(\mathrm{t}, 3 \mathrm{H}, \mathrm{J}=7.3 \mathrm{~Hz}, \mathrm{CH}_{3}-\mathrm{CH}_{2}-\mathrm{C} 2\right), 1.46$ (t, 3H, J=7.3 Hz, $\mathrm{CH}_{3}-\mathrm{CH}_{2}-\mathrm{C} 3$ ). (Found: $\mathrm{C}$, 64.13; $\mathrm{H}, 5.49 ; \mathrm{N}, 7.65 . \mathrm{C}_{19} \mathrm{H}_{19} \mathrm{BrN}_{2}$ requires $\mathrm{C}, 64.23 ; \mathrm{H}, 5.39 ; \mathrm{N}, 7.89$ ).

8b. ${ }^{1}{ }_{\mathrm{H} ~ N M R(C F}$ COOD) $88.91(\mathrm{~s}, 1 \mathrm{H}, \mathrm{H}-1), 8.3-8.15(\mathrm{~m}, 3 \mathrm{H}), 7.9-7.75(\mathrm{~m}, 6 \mathrm{H}), 7.5-7.3(\mathrm{~m}, 3 \mathrm{H}), 5.20(\mathrm{q}, 2 \mathrm{H}, \mathrm{J}=7.1$ $\mathrm{Hz},-\mathrm{CH}_{2} \mathrm{CO}$ ), $1.92\left(\mathrm{t}, 3 \mathrm{H}, \mathrm{J}=7.0 \mathrm{~Hz}, \mathrm{CH}_{3} \mathrm{CH}_{2}\right.$ ). (Found: $\mathrm{C}, 68.00 ; \mathrm{H}, 3.85 ; \mathrm{N}, 5.70, \mathrm{C}_{28} \mathrm{H}_{19} \mathrm{BrN}_{2} \mathrm{O}_{2}$ requires $\mathrm{C}, 67.89$; H, 3.87; N, 5.66).

9a. ${ }^{1} \mathrm{H}$ NMR(CD 3 OD) $88.95(\mathrm{~s}, 1 \mathrm{H}, \mathrm{H}-4), 8.76(\mathrm{~d}, 1 \mathrm{H}, \mathrm{J}=6.9 \mathrm{~Hz}, \mathrm{H}-6), 8.52(\mathrm{~s}, 1 \mathrm{H}, \mathrm{H}-1), 8.43(\mathrm{~d}, 1 \mathrm{H}, \mathrm{J}=6.9 \mathrm{~Hz}, \mathrm{H}-7)$, $8.16(\mathrm{~d}, 1 \mathrm{H}, \mathrm{J}=8.8 \mathrm{~Hz}, \mathrm{H}-8), 7.20(\mathrm{~d}, 1 \mathrm{H}, \mathrm{J}=2.2 \mathrm{~Hz}, \mathrm{H}-11), 7.06(\mathrm{dd}, 1 \mathrm{H}, \mathrm{J}=8.7$ and $2.2 \mathrm{~Hz}, \mathrm{H}-9), 3.97\left(\mathrm{~s}, 3 \mathrm{H}, \mathrm{OCH}_{3}\right)$. $3.06\left(\mathrm{q}, 2 \mathrm{H}, \mathrm{J}=7.3 \mathrm{~Hz}, \mathrm{CH}_{2}-\mathrm{C} 2\right), 2.98\left(\mathrm{q}, 2 \mathrm{H}, \mathrm{J}=7.3 \mathrm{~Hz}, \mathrm{CH}_{2}-\mathrm{C} 3\right), 1.54\left(\mathrm{t}, 3 \mathrm{H}, \mathrm{J}=7.3 \mathrm{~Hz}, \mathrm{CH}_{3}-\mathrm{CH}_{2}-\mathrm{C}_{2}\right), 1.44(\mathrm{t}, 3 \mathrm{H}$, $\mathrm{J}=7.3 \mathrm{~Hz}, \mathrm{CH}_{3}-\mathrm{CH}_{2}-\mathrm{C} 3$ ). (Found: C, 62.15; H, 5.30; N, 7.45. $\mathrm{C}_{20} \mathrm{H}_{21} \mathrm{BrN}_{2} \mathrm{O}$ requires C, 62.34; H, 5.49; N, 7.27). 
9b. ${ }^{1} \mathrm{H}$ NMR(CF 3 COOD) $89.12(\mathrm{~s}, 1 \mathrm{H}, \mathrm{H}-1), 8.6-8.5(\mathrm{~m}, 2 \mathrm{H}), 8.29(\mathrm{~d}, 1 \mathrm{H}, \mathrm{J}=7.1 \mathrm{~Hz}, \mathrm{H}-7), 8.2-8.1(\mathrm{~m}, 2 \mathrm{H}), 8.01(\mathrm{~d}, 1 \mathrm{H}$, $\mathrm{J}=6.9 \mathrm{~Hz}), 7.9-7.7(\mathrm{~m}, 4 \mathrm{H}), 6.95(\mathrm{bd}, 1 \mathrm{H}, \mathrm{H}-9), 5.28\left(\mathrm{q}, 2 \mathrm{H}, \mathrm{J}=7.1 \mathrm{~Hz},-\mathrm{CH}_{2} \mathrm{CO}\right), 3.94\left(\mathrm{~s}, 3 \mathrm{H}, \mathrm{OCH}_{3}\right), 1.94(\mathrm{t}, 3 \mathrm{H}, \mathrm{J}=7.0$ $\mathrm{Hz}, \mathrm{CH}_{3} \mathrm{CH}_{2}$-). (Found: C, $66.15 ; \mathrm{H}, 4.10 ; \mathrm{N}, 5.25 . \mathrm{C}_{29} \mathrm{H}_{21} \mathrm{BrN}_{2} \mathrm{O}_{3}$ requires $\mathrm{C}, 66.29, \mathrm{H}, 4.03 ; \mathrm{N}, 5.33$ ). 10b. ${ }^{1} \mathrm{H}$ NMR(CF $\left.{ }_{3} \mathrm{COOD}\right) 89.12$ (s, $\left.1 \mathrm{H}, \mathrm{H}-1\right), 8.97$ (d, $\left.1 \mathrm{H}, \mathrm{J}=7.1 \mathrm{~Hz}, \mathrm{H}-6\right), 8.42$ (d, $1 \mathrm{H}, \mathrm{J}=7.1 \mathrm{~Hz}$ ), 8.37 (d, $1 \mathrm{H}, \mathrm{J}=7.1$ $\mathrm{Hz}, \mathrm{H}-7), 8.30(\mathrm{~d}, 1 \mathrm{H}, \mathrm{J}=6.8 \mathrm{~Hz}), 8.15-8.05(\mathrm{~m}, 3 \mathrm{H}, \mathrm{H}-8$ and $2 \mathrm{H}$ from the acenaphthene moiety), $7.90(\mathrm{t}, 1 \mathrm{H}, \mathrm{J}=7.5 \mathrm{~Hz})$. $7.83(\mathrm{t}, 1 \mathrm{H}, \mathrm{J}=7.3 \mathrm{~Hz}$ ), 7.7-7.65 (m, 2H, H-10 and H-11), 7.39 (c, $1 \mathrm{H}, \mathrm{J}=6.8 \mathrm{~Hz}, \mathrm{H}-9$ ). (Found: C, $72.70 ; \mathrm{H}, 4.50 ; \mathrm{N}, 7.55$. $\mathrm{C}_{33} \mathrm{H}_{25} \mathrm{~N}_{3} \mathrm{O}_{3} \mathrm{~S}$ requires $\mathrm{C}, 72.91 ; \mathrm{H}, 4.64 ; \mathrm{N}, 7.73$ ).

10c. ${ }^{1} \mathrm{H}$ NMR(CF $\left.{ }_{3} \mathrm{COOD}\right) \delta 9.67$ (s, $\left.1 \mathrm{H}, \mathrm{H}-1\right), 8.97$ (d, $\left.1 \mathrm{H}, \mathrm{J}=7.0 \mathrm{~Hz}, \mathrm{H}-6\right), 8.93$ (d, $\left.1 \mathrm{H}, \mathrm{J}=7.1 \mathrm{~Hz}\right), 8.54$ (d, 1 H, J=7.1 $\mathrm{Hz}$ ), 8.46 (d, 1H, J=7.0 Hz, H-7), 8.29 (d, $2 \mathrm{H}, \mathrm{J}=7.1 \mathrm{~Hz}$ ), 8.14 (d, 1H, J=7.2 Hz, H-8), 7.81 (L, 1H, J=7.2 Hz), 7.75-7.6 ( $\mathrm{m}, 5 \mathrm{H}, \mathrm{H}-10, \mathrm{H}-11$ and $3 \mathrm{H}$ from the phenanthrene moiety), $7.44(\mathrm{t}, 1 \mathrm{H}, \mathrm{J}=6.8 \mathrm{~Hz}, \mathrm{H}-9$ ). (Found: $\mathrm{C}, 73.50 ; \mathrm{H}, 4.65 ; \mathrm{N}$, 7.20. $\mathrm{C}_{35} \mathrm{H}_{27} \mathrm{~N}_{3} \mathrm{O}_{3} \mathrm{~S}$ requires $\mathrm{C}, 73.79 ; \mathrm{H}, 4.78 ; \mathrm{N}, 7.38$ ).

11b. ${ }^{1} \mathrm{H}$ NMR(CF 3 COOD) 89.13 (s, 1H, H-1), 9.03 (d, 1H, J=7.1 Hz, H-6), 8.49 (d, 1H, J=7.1 Hz, H-7), 8.37 (d, 1H, $\mathrm{J}=6.8 \mathrm{~Hz}, 2 \mathrm{H}), 8.24$ (d, $1 \mathrm{H}, \mathrm{J}=8.3 \mathrm{~Hz}), 8.19(\mathrm{~d}, 1 \mathrm{H}, \mathrm{J}=8.5 \mathrm{~Hz}), 8.09$ (d, $1 \mathrm{H}, \mathrm{J}=9.0 \mathrm{~Hz}, \mathrm{H}-8), 8.0-7.9(\mathrm{~m}, 2 \mathrm{H}), 7.23(\mathrm{~s}, 1 \mathrm{H}$, $\mathrm{H}-11$ ), 7.09 (d, $1 \mathrm{H}, \mathrm{J}=8.9 \mathrm{~Hz}, \mathrm{H}-9), 3.99\left(\mathrm{~s}, 3 \mathrm{H}, \mathrm{OCH}_{3}\right.$ ). (Found: C, $71.20 ; \mathrm{H}, 4.50, \mathrm{~N}, 7.60 \mathrm{C}_{34} \mathrm{H}_{27} \mathrm{~N}_{3} \mathrm{O}_{4} \mathrm{~S}$ requires $\mathrm{C}$, 71.18; $\mathrm{H}, 4.74 ; \mathrm{N}, 7.33)$.

11c. ${ }^{1} \mathrm{H}$ NMR(CF ${ }_{3}$ COOD) 89.42 (s, $\left.1 \mathrm{H}, \mathrm{H}-1\right), 8.9-8.8(\mathrm{~m}, 2 \mathrm{H}), 8.47$ (d, $\left.1 \mathrm{H}, \mathrm{J}=7.5 \mathrm{~Hz}\right), 8.3-8.2(\mathrm{~m}, 3 \mathrm{H}), 7.9-7.7$ (m, $\left.5 \mathrm{H}\right)$, 7.0-6.9 (m, 2H, $\mathrm{H}-9$ and $\mathrm{H}-11), 3.85\left(\mathrm{~s}, 3 \mathrm{H}, \mathrm{OCH}_{3}\right.$ ). (Found: $\mathrm{C}, 71.95 ; \mathrm{H}, 4.90 ; \mathrm{N}, 6.85 \mathrm{C}_{36} \mathrm{H}_{29} \mathrm{~N}_{3} \mathrm{O}_{4} \mathrm{~S}$ requires $\mathrm{C}, 72.10$; H, 4.87; N, 7.01).

8. Prasad, K. B.; Swan G. A. J. Chem. Soc. 1958, 2024.

9. Matia, M.P.; Garcia-Navio, J.L.; Vaquero, JJ.; Alvarez-Builla, J. J. Heterocyclic Chem. 1990, $27,661$.

10. Standard procedure: To a stirred solution of O-mesitylenesulfonylhydroxylamine (MSH) $(2.15 \mathrm{~g}, 10 \mathrm{mmol})$ in dichloromethane $(20 \mathrm{ml})$, the corresponding arine $(10 \mathrm{mmol})$ in the same solvent $(20 \mathrm{ml})$ was dropwise added. The mixture was stirred at room temperature for $10 \mathrm{~min}$. Diethyl ether $(30 \mathrm{ml})$ was then added to precipitate the $\mathrm{N}$ aminoazinium salts 6 and 7 which were triturated with ether $(3 \times 5 \mathrm{ml})$ and recrystallized from ethanol.

6. $\left(217-219^{\circ} \mathrm{C}, 92 \%\right){ }^{1} \mathrm{H}$ NMR(CD $\left.{ }_{3} \mathrm{OD}\right) 88.44$ (d, $\left.1 \mathrm{H}, \mathrm{J}=6.7 \mathrm{~Hz}, \mathrm{H}-3\right), 8.37$ (d, $\left.1 \mathrm{H}, \mathrm{J}=6.8 \mathrm{~Hz}, \mathrm{H}-4\right), 8.30$ (d, $1 \mathrm{H}, \mathrm{J}=8.1$ $\mathrm{Hz}, \mathrm{H}-5$ ), 7.8-7.7 (m, 2H, H-7 and H-8), 7.5-7.4 (m, 1H, H-6), 3.09 (s, 3H, $\mathrm{CH}_{3}-\mathrm{Cl}$ ). (Found: C, 63.25; H, 5.90; N, 10.77. $\mathrm{C}_{21} \mathrm{H}_{23} \mathrm{~N}_{3} \mathrm{O}_{3} \mathrm{~S}$ requires $\mathrm{C}, 63.45 ; \mathrm{H}, 5.83 ; \mathrm{N}, 10.57$ ).

7. $\left(234-236^{\circ} \mathrm{C}, 87 \%\right.$ ) ${ }^{1} \mathrm{H}$ NMR(DMSO-d $\left.\mathrm{d}_{6}\right) \delta 12.60(\mathrm{~s}, 1 \mathrm{H}, \mathrm{NH}), 8.46(\mathrm{~d}, 1 \mathrm{H}, \mathrm{J}=6.9 \mathrm{~Hz}, \mathrm{H}-3), 8.36$ (d, $1 \mathrm{H}, \mathrm{J}=6.9 \mathrm{~Hz}, \mathrm{H}-$ 4), $8.26(\mathrm{~d}, 1 \mathrm{H}, \mathrm{J}=8.5 \mathrm{~Hz}, \mathrm{H}-5), 7.65\left(\mathrm{~s}, 2 \mathrm{H}, \mathrm{NH}_{2}\right), 7.10$ (d, $\left.1 \mathrm{H}, \mathrm{J}=2.0 \mathrm{~Hz}, \mathrm{H}-8\right), 7.01$ (dd, $1 \mathrm{H}, \mathrm{J}=8.4$ and $1.9 \mathrm{~Hz}, \mathrm{H}-6$ ), 3.91 (s, 3H, OCH 3 ). 2.97 (s, 3H, CH 3 -Cl). (Found C, 62.00; $\mathrm{H}, 5.70 ; \mathrm{N}, 9.80 . \mathrm{C}_{22} \mathrm{H}_{25} \mathrm{~N}_{3} \mathrm{O}_{4} \mathrm{~S}$ requires $\mathrm{C}, 61.80 ; \mathrm{H}$, $5.89 ; \mathrm{N}, 9.83$ ).

11. Authors wish to acknowledge the assistance provided by LILLY S.A. for the Nmr analysis, and to the Comision Interministerial de Ciencia y Tecnologia (CICYT) for financial support (Project PB87-0755). 